\title{
Systems biology approaches for analysis of dementia with Lewy bodies in mouse models
}

\author{
T.G. Amstislavskaya ${ }^{1,4 *}$, M.A. Tikhonova ${ }^{1,4}$, H. Bai ${ }^{3}$, Y.L. Orlov ${ }^{2,4}$, M. Chen ${ }^{3,5}$ \\ ${ }^{1}$ Scientific Research Institute of Physiology and Basic Medicine, Novosibirsk, Russia \\ ${ }^{2}$ Institute of Cytology and Genetics SB RAS, Novosibirsk, Russia \\ ${ }^{3}$ Inner Mongolia University for the Nationalities, China \\ ${ }^{4}$ Novosibirsk State University, Novosibirsk, Russia \\ ${ }^{5}$ Zhejiang University, Hangzhou, China \\ *e-mail: amstislavskayatg@physiol.ru
}

Key words: neurodegenerative diseases, dementia with Lewy bodies, mouse models, systems biology

Motivation and Aim: We present joint research project dedicated to the fundamental problem of neurophysiology and metabolism related to the elucidation of the physiological and molecular mechanisms of the disorders caused by aging and neurodegenerative processes. The estimated economic burden caused by dementia related problems was US\$ 18 billion in 2015 and would rise above \$1 trillion by 2018 . The common causes of dementia in elderly are the Alzheimer's disease, vascular dementia and dementia with Lewy bodies (DLB). Our aim was to identify the opportunities to correct these neurological pathologies and their related dysfunctions using experimental molecularbiological and bioinformatics approaches.

Methods and Algorithms: The dementia has complex genetic background and shares high comorbidity with metabolic diseases, diabetes, bipolar disorder, and alcohol addiction, thus presenting socially important problem in Asian populations. There is evidence of multi-factorial nature of these diseases. Based on population genetics analysis and network models we select target genes regulating the diseases networks. We validate gene targets experimentally as specific candidates for combined treatment. To understand the genetic etiology of major neurodegenerative processes we model dementia with Lewy bodies in mice to test drug effects on gene targets.

Results: We created original experimental model of DLB based on the combined effects of $\alpha$-syn and A $\beta$. Mice of transgenic B6.Cg-Tg (Prnp-SNCA*A53T)23Mkle/J strain overexpressing a mutant form of the human $\alpha$-syn were administered with $A \beta$ into the lateral ventricles of the brain. We used several steps of systems biology analysis concluded by construction of experimental model in mouse. The original experimental models of various forms of proteinopathy associated with the pathogenesis of DLB will be used to study the immune characteristics and the severity of neuro-inflammation in different brain areas that accompany neurodegeneration.

Conclusion: The results broaden the modern notion about the role of neuro-inflammation in the pathogenesis of DLB as well as to evaluate the prognostic significance of the concentrations of pro-inflammatory cytokines (TNF-alpha, IL-1, IL-6) in the blood as biomarkers for the differential diagnosis of dementia with proteinopathy.

Acknowledgements: The research has been supported by Russian RFBR, Indian DST and Chinese NSCF. Computing was supported by ICG SB RAS budget project 0324-20180017. 